main, has been found only in the hypothalamus. NPY is the most abundant neuropeptide in the brain, and is a member of a family of proteins, including pancreatic polypeptide (PP), peptide YY (PYY) and seminalplasmin, that are characterized by a proline helix folded onto an $\alpha$ helix (pancreatic polypeptide fold) with a carboxy-terminal tyrosine. The region of the hypothalamus involved in feeding has been reported to have the Y1, Y2, PP1 and perhaps a unique 'feeding' receptor. These receptors and peptides can crossreact, implying that the other peptides could replace NPY.

Physiologically, NPY seems to have various functions ${ }^{6,7}$ - including involvement in regulation of circadian rhythm, anxiety and stress response, sexual function, peripheral vascular resistance and cardiac contractility, as well as of food intake and carbohydrate preference, and metabolic and lipogenic rate. Various lines of evidence show that NPY is the most effective neuropeptide in inducing feeding behaviour. Levels of the peptide and its messenger RNA correlate with the normal feeding cycle in rodents, and NPY neurons occur in the appropriate areas of the hypothalamus ${ }^{8,9}$. When injected centrally, antibodies and antisense oligonucleotides directed against NPY block normal onset of feeding. And NPY is the only known peptide that can induce obesity through prolonged central administration.

Erickson et al. ${ }^{1}$ have produced an important animal model for studying the role of NPY in the long-term regulation of feeding and adiposity. In contrast to the acute intervention studies cited above, they report that the onset of feeding is normal following fasting in $-1-$ mice. The response to leptin is intact and even more pronounced than in $+/+$ mice: leptin promotes an exaggerated and immediate inhibition of food intake in $-/-$ mice that tends to abate with time, whereas the response in $+/+$ animals is slow, gradually increasing for days. Is the response to leptin in $-/-$ mice physiological or the result of abnormalities arising from NPY deficiency? How does feeding behaviour and metabolism in $-/-$ animals respond to nutrient excess, nutrient deficit or leptin administration for a time sufficient to alter their adipos-

1. Erickson, J. C., Clegg, K. E. \& Palmiter, R. D. Nature 381, 415-418 (1996)

2. Zhang, Y. et al. Nature 372, 425-432 (1994)

3. Stephens, T. W. et al. Nature 377, 530-532 (1995).

4. Schwartz, M. W. et al. Diabetes 45, 531-535 (1996).

5. Gwo-Hwa, L. et al. Nature 379, 632-635 (1996).

6. White, J. D. Reg. Peptides 49, 93-107 (1993).

7. Leibowitz, S. F. Ann. N. Y. Acad. Sci. 31, 12-35 (1994).

8. Davies, L. \& Marks, J. L. Am. J. Physiol. 266, R1687-R1691 (1994).

9. Frankish, H. M., Dryden, S., Hopkins, D., Wang, Q. \& Williams, G. Peptides 16, 757-771 (1995). ity? Cross-breeding with mice having known defects in body-weight regulation may give clues to the answers to these questions.

The release of NPY in the hippocampus has been referred to as that of an 'endogenous anticonvulsant' because of its inhibition of presynaptic excitatory glutamate release ${ }^{10}$. This inhibition is mediated by presynaptic $\mathrm{Y} 2$ receptors, which appear to be the predominant site for NPY action in this region of the brain. So Erickson and colleagues' observation of the propensity for $-/-$ mice to have seizures, when under mild stress or after administration of an antagonist to the inhibitory neurotransmitter GABA $(\gamma$ aminobutyric acid), is an important confirmation that NPY participates in the inhibition of hippocampal glutamate release. Studies of other behavioural abnormalities expected with the $-/-$ mutation could lead to further insight into NPY regulation of neurotransmitter release.

Finally, what about the question posed at the outset? Does the lack of observed abnormality in feeding in $-1-$ mice indicate the existence of compensatory mechanisms, or does NPY play a more complex part in the regulation of feeding than we think? Targeted disruption of a member of a family of proteins can indeed result in compensation by other members ${ }^{11,12}$. Knockout of several brain proteins results in different phenotypes from those predicted from classical pharmacology, and immunolocalization, antisense oligonucleotide, and immunoneutralization studies $^{13-17}$. These knockouts reveal subtle abnormalities that are at odds with the hypothesized role of NPY based on acute intervention studies.

So NPY may be one component in a rather plastic circuitry that features redundant molecular mechanisms and can withstand the loss of some of them. Selective knockout of NPY in specific locations of the hypothalamus, perhaps with genes that are upregulated in the mature animal, will probably be required to further our knowledge of the physiological action of NPY.

Thomas W. Stephens is at Lilly Research Laboratories, Lilly Corporate Center, Indianapolis, Indiana 46226, USA.

10. Colmers, W. \& Bleakman, D. Trends Neurosci. 17 373-379 (1994).

11. Hummler, E. et al. Proc. natn. Acad. Sci. U.S.A. 91, 5647-5651 (1994).

12. Araki, E. et al. Nature 372, 186-190 (1994).

13. Strittmatter, S. M., Fanhauser, C., Huang, P. L., Mashimo, H. \& Fishman, M. C. Cell 80, 445-452 (1995).

14. Abellovich, A. et al. Cell 75, 1253-1262 (1993)

15. Grant, S. G. N. et al. Science 258, 1903-1910 (1992).

16. Silva, A. J., Paylor, R., Wehner, J. M. \& Tonegawa, S. Science 257, 206-211 (1992)

17. Conquet, F. et al. Nature 372, 237-243 (1994)
DAEDALUS

\section{Film for ever!}

THE modern information age will disappoint historians. Our paper, made from acid wood-pulp, will decay in less than a century; our magnetic disks and cassette tapes will embrittle and peel, even if ceaseless changes of format leave any machines capable of playing them; the dyes in our colour films and photographs will fade to a drab beige. Only monochrome photographs, their metallic silver image stably held in crosslinked gelatine, will survive. Most of our so-called information is worthless trivia and is best forgotten anyway; but Daedalus would like to save something from its wreckage. He is devising an archival digital colour film.

Current colour films are 'analogue subtractive' systems. Incident light penetrates a series of coloured layers, each of which absorbs and subtracts a specific waveband. By contrast, a shadow-mask television tube is 'digital additive'. Red, green and blue dots of phosphor form a hexagonal matrix on the screen, are addressed as pixels by the electron beams, and their light output is added together.

At first Daedalus hoped to copy this additive architecture by printing a pattern of red, green and blue filter dots on a monochrome emulsion. Blue light (say) would activate the halide grains under the blue dots. Processing would block these dots with black developed silver, leaving the red and green dots free to transmit their sum - yellow, the complementary hue of the colour-negative.

But a true digital film should address the tiny halide grains as individual pixels. So Daedalus must line them up into an addressable array. He will exploit the fact that silver halides are diamagnetic. In a strong magnetic field, their grains repel each other. Expose the emulsion to such a field while it is still liquid, and its grains will adopt the arrangement of maximum repulsion - a perfect hexagonal array. The pattern of coloured dots, defining red-, green- and blue-sensitive grains, can then be printed on top in register.

Of course, the dyes in those dots will be just as fugitive as the ones in conventional film. Digital negatives and prints will fade as surely, but will leave a stable silver monochrome image behind. Furthermore, each grain will be digitally colour-coded by its position in the original matrix. A historian with a microscope could identify red, green and blue grains, and reconstruct the coloured image. Alone among our media, digital colour film will survive. Our age will shine down the centuries in all its vacuous chromatic splendour, but with no word of explanation. David Jones 